\title{
Myocardial bridging presenting as an acute coronary syndrome with mitral valve stenosis and paroxysmal atrial fibrillation: A case report
}

\author{
Maureen Victoria Kawilaranga, Dita Aulia Rachmia, Yusuf Azmia, Mohammad \\ Budiarto $^{\mathrm{a}}$ \\ a maureenvictoria88@gmail.com \\ ${ }^{a}$ Department of Cardiology and Vascular Medicine, Faculty of Medicine, Universitas Airlangga - Dr. Soetomo General Hospital, \\ Surabaya 60286, Indonesia
}

\begin{abstract}
We report a 33-year-old man presented to the emergency department with a chief complaint of typical angina three days before admission. An electrocardiogram showed arrowhead T-waves inversion in leads V1-V4, and the cardiac enzyme marker was increased. The initial diagnosis was non-ST-elevation acute coronary syndrome. On subsequent examinations, he had severe mitral stenosis, paroxysmal atrial fibrillation, and a history of intracranial hemorrhage. Diagnostic coronary angiography examination revealed myocardial bridging in the middle segment of left anterior descending coronary arteries. The patient was given dual antiplatelets (DAPT), beta-blocker, atorvastatin, and anticoagulant. Beta-blocker is the first-line therapy for myocardial bridging, whereas DAPT, anticoagulant, and high-dose statin therapy are used to treat acute coronary syndrome (ACS). The atherosclerotic process is commonly found in the proximal segment of myocardial bridging that can lead to ACS. In addition, mitral stenosis is one of the main causes of systemic embolism, including coronary embolism, while atrial fibrillation is known to increase the risk of embolism. Herein, we discuss the possible etiology of ACS, the management of myocardial bridging and comorbidity of this patient, and the rationale for selecting antithrombotic therapy.
\end{abstract}

Keywords: Myocardial bridging; acute coronary syndrome; mitral stenosis; atrial fibrillation

\section{Introduction}

Acute coronary syndrome (ACS) is the leading cause of death worldwide, characterized by chest pain with or without ST-segment and T-wave changes on the electrocardiogram (ECG) and with or without elevated cardiac enzymes. ACS includes unstable angina pectoris, acute non-ST-segment elevation coronary syndrome (NSTEACS), and ST-segment elevation myocardial infarction (STEMI) (Thygesen et al., 2012). It is estimated that of all patients diagnosed with acute myocardial infarction (AMI) who underwent coronary angiography or autopsy in deceased patients, about 4-7\% do not have significant atherosclerosis (Kolodgie et al., 2015). Mitral stenosis is one of the main causes of systemic embolism, including coronary embolism, while atrial fibrillation is known to increase the risk of embolism (Cardoz et al., 2015).

Myocardial bridging is a congenital coronary abnormality of the intramyocardial segment of the epicardial coronary arteries lined by cardiac muscle, which causes compression of the arteries during systole and, in most cases, are asymptomatic. Generally, the myocardial bridging is confined to the middle of the left anterior descending (LAD) artery. Diagnostic coronary angiography (DCA) and intravascular ultrasonography (IVUS) can document typical disturbances of systolic flow. The atherosclerotic process is more commonly found in the proximal segment of myocardial bridging that can lead to the incidence of ACS (Alegria et al., 2005). Betablockers, calcium channel blockers, ivabradine, and vasodilating agents such as nitroglycerin are used in the management of myocardial bridging (Murtaza et al., 2020). Percutaneous coronary intervention (PCI), coronary artery bypass graft (CABG), and surgical myotomy may be considered in patients with refractory to medical therapy and a high risk of acute cardiac manifestations (Tsujita et al., 2009; Wu and Xu, 2007).

\section{Case Report}

A 33-year-old man presented to the emergency department with a chief complaint of typical angina three days before admission. The patient had a history of mitral stenosis, hemorrhagic stroke, and ischemic stroke two years 
ago. The patient routinely took furosemide, spironolactone, bisoprolol, digoxin, and warfarin. He has smoked for 20 years.

On physical examination, the pain scale was 5/10, blood pressure was 109/63 $\mathrm{mmHg}$, heart rate was 60 beats $/ \mathrm{min}$, and respiratory rate was 20 times/min. Heart auscultation revealed a diastolic murmur at the apex with a scale of III/VI and extrasystole. Electrocardiogram (ECG) showed arrowhead T-waves inversion in leads V1-V4 and multiple atrial extrasystoles (Figure 1). Transthoracic echocardiography (TTE) revealed severe mitral stenosis and left atrial dilatation (LA major $7.9 \mathrm{~cm}$ ). No intracardiac thrombus or vegetation was observed, but left atrial spontaneous echo contrast (LASEC) was positive. The left ventricle (LV) was normokinetic and normal systolic function with an ejection fraction of $65 \%$. There was no LV hypertrophy. Right ventricle (RV) systolic function was normal (TAPSE $2.4 \mathrm{~cm}$ ). On laboratory examination, the cardiac enzyme marker was increased with CKMB $46 \mathrm{ng} / \mathrm{mL}$ and troponin-I 0,036 (cut off 0,01).

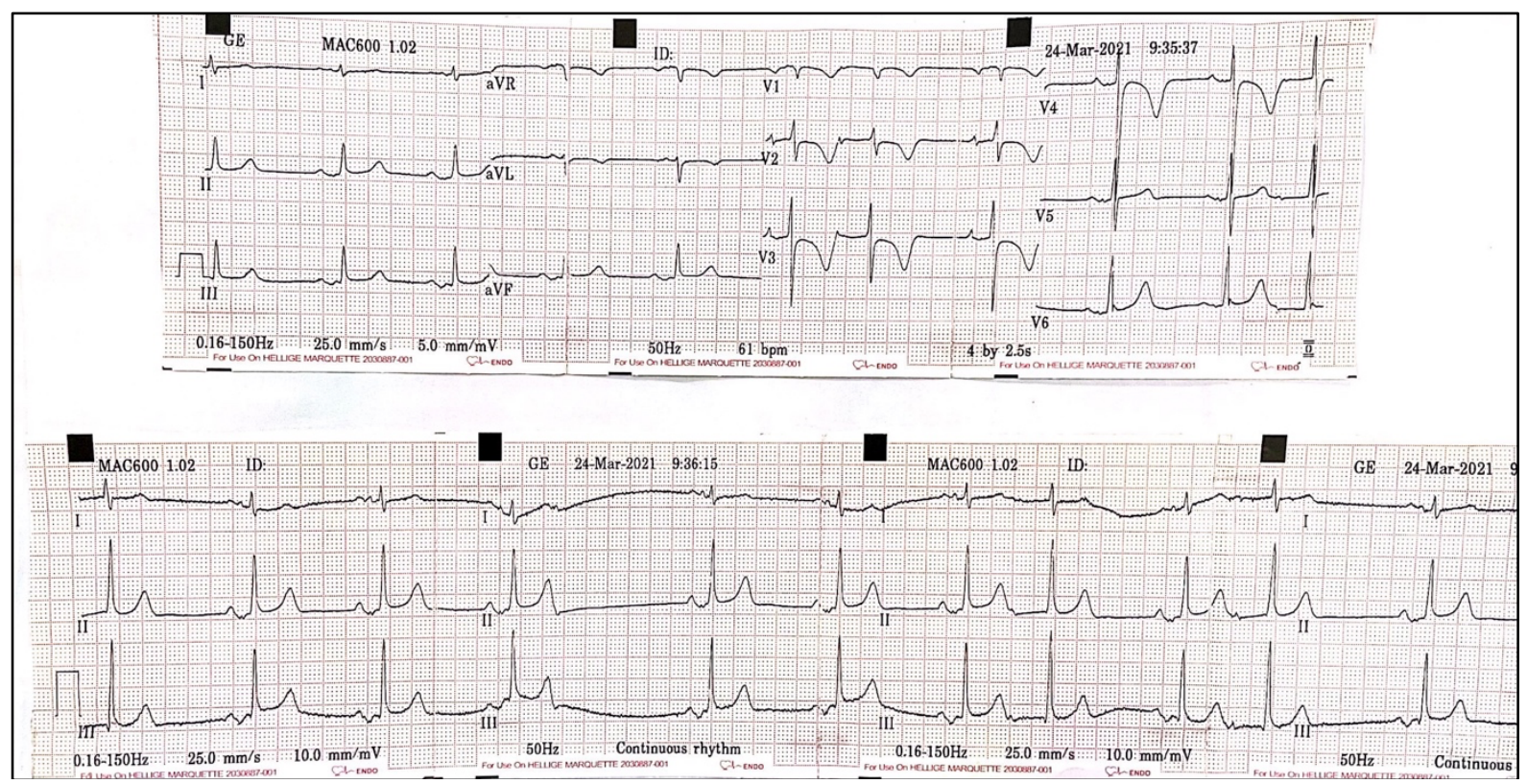

Figure 1. Electrocardiography showed arrowhead $\mathrm{T}$ wave-inversion in leads V1-V4 and multiple atrial extrasystoles.

The initial diagnosis was non-ST-elevation acute coronary syndrome (NSTEACS). The patient was given aspirin $300 \mathrm{mg}$ loading dose, clopidogrel $300 \mathrm{mg}$ loading dose, bisoprolol $2.5 \mathrm{mg}$, atorvastatin $40 \mathrm{mg}$, and enoxaparin 60 $\mathrm{mg}$ subcutaneous injection. The patient developed paroxysmal atrial fibrillation (Figure 2) and hematuria during hospitalization. Since there was no significant decrease in hemoglobin, the dual antiplatelet (DAPT) regimen was changed to clopidogrel and warfarin. 


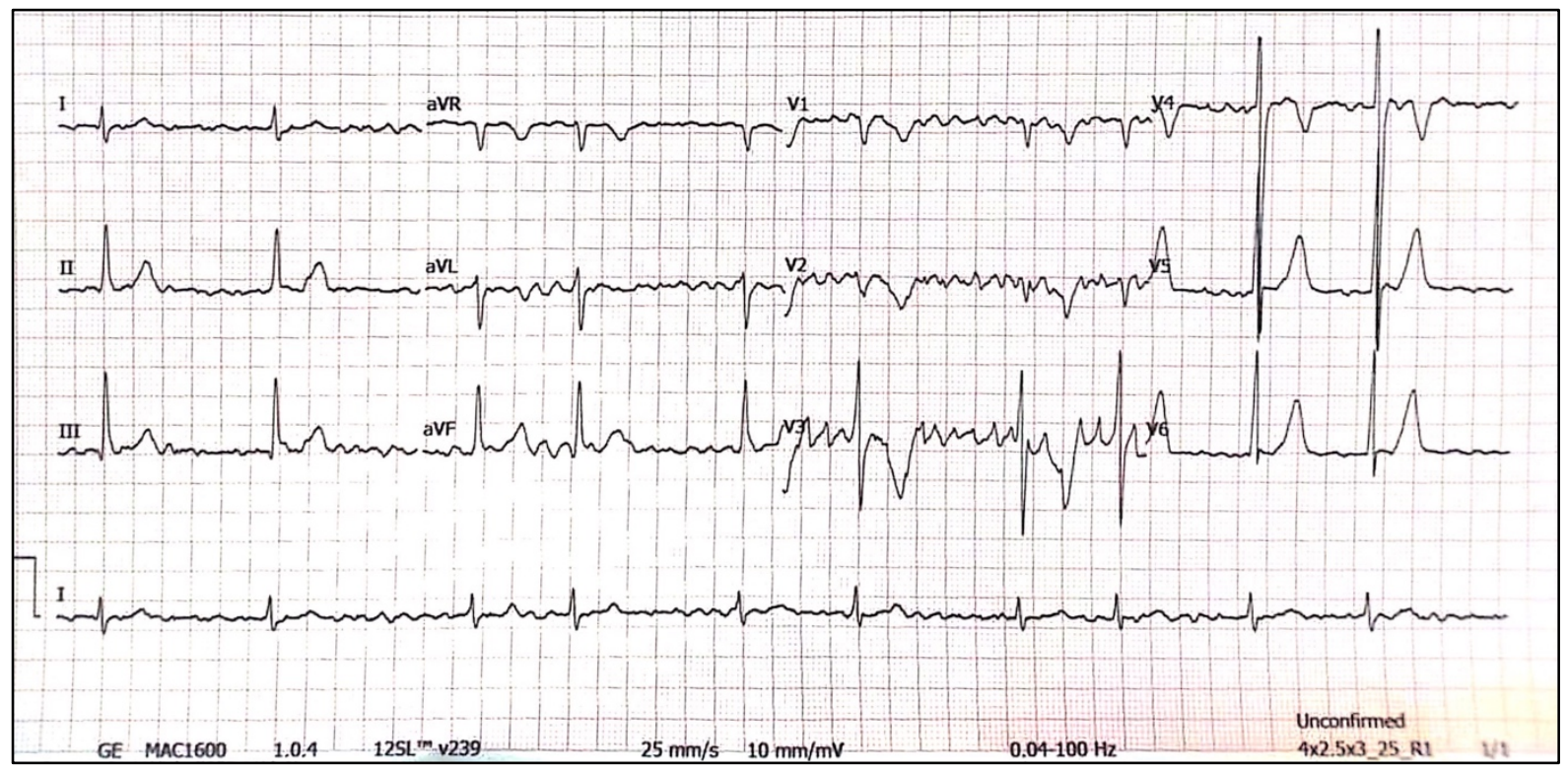

Figure 2. Paroxysmal atrial fibrillation rhythm during hospitalization.

Diagnostic coronary angiography (DCA) examination revealed myocardial bridging in the middle segment of left anterior descending (LAD) coronary arteries. No abnormality was observed in the right coronary artery (RCA), left main coronary artery (LMCA), and left circumflex artery (LCx) (Figure 3a-d). Symptom of angina was reduced significantly after medical therapy to a scale of $1 / 10$. After the seventh day of hospitalization, the patient was discharged and followed up at the outpatient clinic.

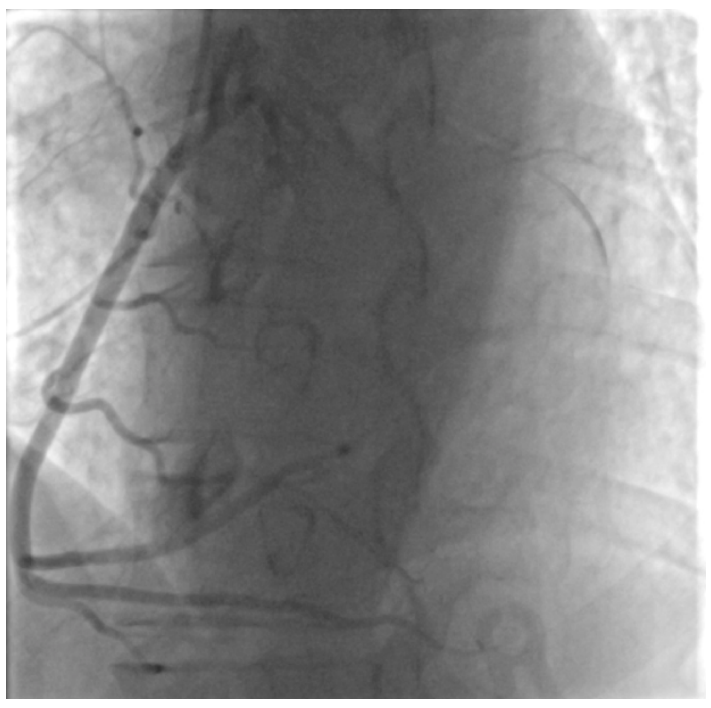

(a)

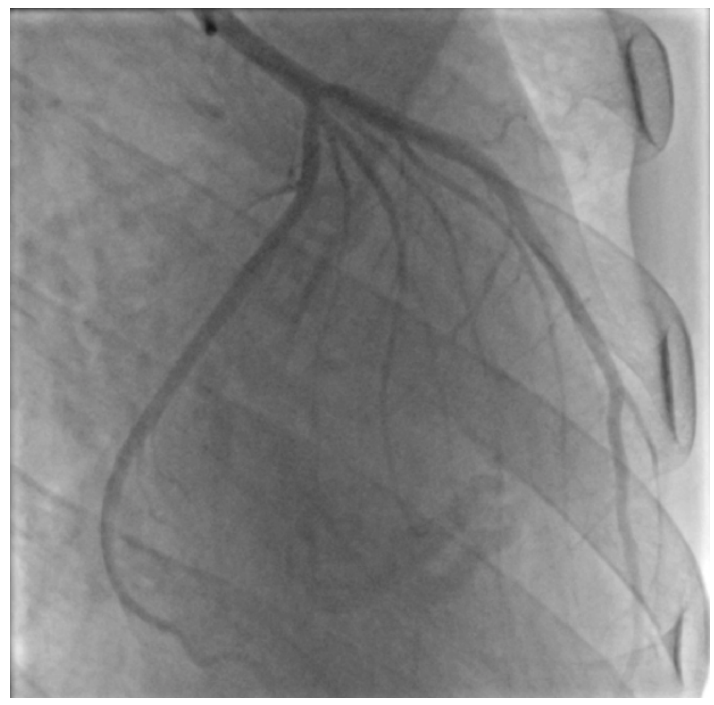

(b) 


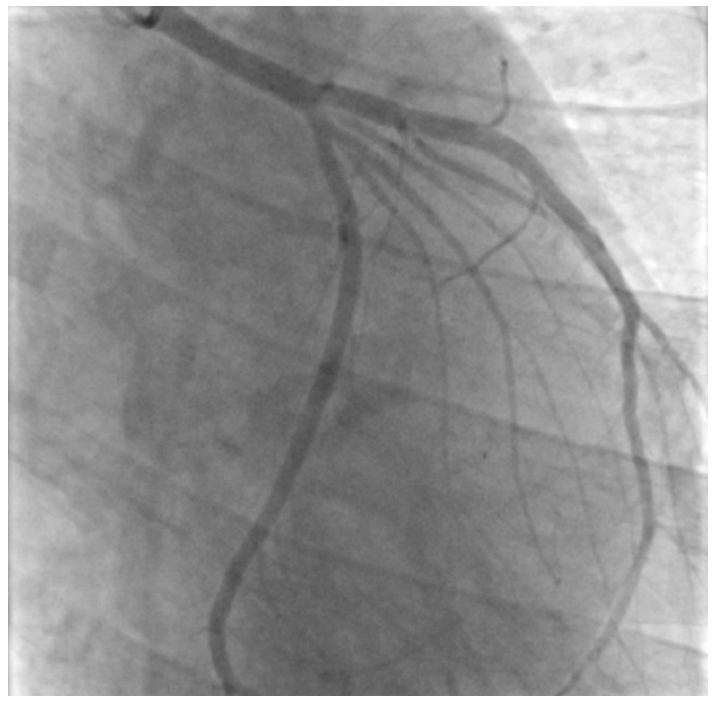

(c)

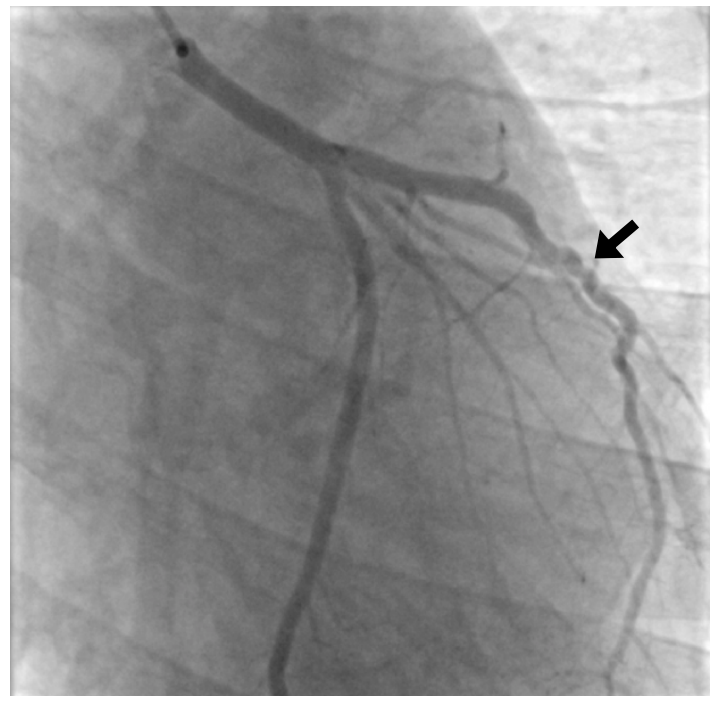

(d)

Figure 3. Normal coronary angiography for (a) right coronary artery, (b) left circumflex coronary artery, (c) left anterior descending coronary during diastole, and (d) myocardial bridging in the middle segment of the left anterior descending coronary artery during systole (arrowhead sign).

\section{Discussion}

We report a patient with myocardial bridging with ACS presentation. In addition, patients had a history of mitral stenosis and hemorrhagic stroke two years ago, and paroxysmal atrial fibrillation was developed during hospitalization. Herein, we discuss the possible causes of ACS, the management of myocardial bridging and comorbidity of this patient, and the rationale for selecting antithrombotic therapy.

Myocardial bridging is an intramyocardial segment of an epicardial coronary artery lined by cardiac muscle, whereas the tunnel artery refers to arteries that flow within the myocardium. This condition results in compression of the tunnel artery during systole, which in most cases are asymptomatic (Möhlenkamp et al., 2002). Myocardial bridging may be single or multiple, often localized in the middle segment of the LAD, followed by diagonal and marginal branches (Yuan, 2016). Myocardial bridging is divided into two types, including superficial muscle type and deep muscle type. The superficial type obstructs blood flow during systole, while the deep muscle type reduces blood flow and causes myocardial ischemia (Ferreira et al., 1991). Another division divides myocardial bridging into three classes, including grade one with less than 50\% narrowing, grade two with $50 \%$ to $75 \%$ narrowing, and grade three with more than $75 \%$ narrowing (Noble et al., 1976).

Although usually small and not clinically significant, the myocardial bridging is capable of causing ischemia. The atherosclerotic process is more commonly found in the proximal segment than in the myocardial bridging segment. Impaired flow and high blood pressure, changes in endothelial morphology due to mechanical stress, increased vasoactive agents, and platelet aggregation can lead to the incidence of ACS (Alegria et al., 2005). The incidence of myocardial ischemia in myocardial bridging ranges from $21 \%$ to $88 \%$, with clinical manifestations varying from angina, ACS, LV dysfunction, ventricular arrhythmias to sudden cardiac death (Centurión et al., 2019). These manifestations develop from compression of the tunnel segment due to myocardial contraction or atherosclerotic stimulation in the proximal segment of the myocardial bridging (Yuan, 2016).

The diagnosis of myocardial bridging can generally be established by DCA, where coronary artery narrowing is found during systole, and atherosclerotic lesions are often found in the proximal segment. When DCA results are uncertain, IVUS can be used for confirmation because it has a higher sensitivity value and can be combined with provocation tests with nitroglycerin, dobutamine, and acetylcholine (Lee and Chen, 2015). As the mainstay of conservative treatment, beta-blockers can reduce heart rate, thereby reducing the risk of hemodynamic compromise. In patients who cannot tolerate beta-blockers, calcium channel blockers (CCB) with vasodilating effects may be beneficial. In symptomatic patients who cannot tolerate beta-blockers/CCBs, ivabradine can be given as a second-line treatment. Vasodilating agents such as nitroglycerin should be used with caution and only 
indicated in the presence of significant coronary vasospasm because it can vasodilate the proximal segment of the myocardium and increase systolic compression. Patients with myocardial bridging with subclinical atherosclerosis should receive antiplatelet agents to reduce future adverse cardiac events (Murtaza et al., 2020). Percutaneous coronary intervention should be considered in patients who are refractory to medical therapy and have a high revascularization potential with drug-eluting stents (Tsujita et al., 2009). In addition, surgical approaches such as coronary artery bypass graft (CABG) and surgical myotomy may be considered. Anastomosis of the left internal mammary artery to the LAD artery is commonly performed in CABG. Surgical myotomy is reserved for patients refractory to medical therapy and demonstrates induced ischemia, risk of ventricular tachycardia, and sudden cardiac death (Wu and $\mathrm{Xu}, 2007)$.

The risk of ACS increased in patients with mitral stenosis, where a thrombus that forms in the left atrium may embolize systemic arteries, including the coronary arteries, although this event is rare. Thus, the incidence of myocardial infarction (MI) in this patient, apart from being caused by myocardial bridging, may also be due to coronary embolization from thrombus formed in the left atrium (Cardoz et al., 2015; Chaurasia et al., 2013). The presence of paroxysmal AF in this patient may also be a trigger for ACS. Patients with AF have an increased risk of MI which is estimated to be due to coronary thromboembolism (Soliman et al., 2014). The occurrence of AFassociated thromboembolism may be explained by Virchow's triad consisting of blood stasis, hypercoagulable state, and endothelial damage (Henriques et al., 2002). In addition, AF stimulates thrombosis through elevated levels of inflammatory markers (Iwasaki et al., 2011).

Patients with indications for "triple" antithrombotic therapy, as in ACS patients with AF, may have a dilemma because of uncertainty about the ischemic consequences and bleeding risk of eliminating one antiplatelet agent or reducing the dose of anticoagulant. A study with warfarin plus clopidogrel produced a nonsignificant effect on the reduction of major bleeding compared with triple antiplatelet therapy, without increasing thromboembolic events (Dewilde et al., 2013). This report underpinned our decision to switch the DAPT regimen to clopidogrel and warfarin. In addition, Acute Coronary Syndrome or PCI in the Atrial Fibrillation Trial (AUGUSTUS) showed that in $23 \%$ of enrolled patients with ACS who received medical treatment showed that oral anticoagulation (OAC) with apixaban significantly reduced bleeding incidence compared with vitamin $\mathrm{K}$ antagonists (VKA) (Lopes et al., 2019). Another study examined the use of very low-dose rivaroxaban ( $2.5 \mathrm{or} 5 \mathrm{mg}$ twice daily) in patients with a recent ACS receiving DAPT found that the addition of rivaroxaban significantly reduced the risk of cardiovascular death, MI, or stroke but increased the risk of bleeding (Mega et al., 2012).

The history of intracranial hemorrhage with atrial fibrillation in mitral stenosis, in this case, warrants special consideration in the administration of antiplatelets and anticoagulants. An increased risk of anticoagulantassociated intracranial hemorrhage $(\mathrm{AICH})$ was observed in patients with thromboembolic disease receiving anticoagulant therapy. Especially with warfarin, when the INR is between $2-4.5$, the risk of AICH is 0.3 to $3.7 \%$ (Ray and Keyrouz, 2014). Due to the high risk of anticoagulation which can cause hematoma expansion and high morbidity due to warfarin-related intracerebral hemorrhage (WRICH), anticoagulation is recommended to be temporarily discontinued during the acute phase of $\mathrm{ICH}$. Anticoagulation may be considered for re-administration 10 to 14 days post-WRICH with close monitoring (Steiner, 2011).

\section{Conclusion}

We report a case of myocardial bridging presenting as an acute coronary syndrome. This patient also had mitral valve stenosis and paroxysmal AF, which may be a trigger for ACS. In addition, the history of hemorrhagic stroke makes the comprehensive anticoagulant management in this patient urgently needed to make a better outcome. Additional studies are needed to assess the best therapy in such cases. 


\section{References}

Alegria, J.R., Herrmann, J., Holmes Jr, D.R., Lerman, A., Rihal, C.S., 2005. Myocardial bridging. Eur. Heart J. 26, 1159-1168.

Cardoz, J., Jayaprakash K, D., George, R., 2015. Mitral stenosis and acute ST elevation myocardial infarction, in: Baylor University Medical Center Proceedings. Taylor \& Francis, pp. 207-209.

Centurión, O.A., Alderete, J.F., Torales, J.M., 2019. Myocardial bridge as a cause of myocardial ischemia and infarction:"Bridge over troubled waters" and turbulent coronary blood flow. J Cardiol Curr Res 12, 136140.

Chaurasia, A.K., Harikrishnan, S., Ajith, V.K., Tharakan, J.M., 2013. Mitral Stenosis, LV Aneurysm, Myocardial Bridge, and Myocardial Infarction: The Mystery Demystified. Int. Cardiovasc. Res. J. 7, 104.

Dewilde, W.J.M., Oirbans, T., Verheugt, F.W.A., Kelder, J.C., De Smet, B.J.G.L., Herrman, J.-P., Adriaenssens, T., Vrolix, M., Heestermans, A.A.C.M., Vis, M.M., Tijsen, J.G.P., van 't Hof, A.W., ten Berg, J.M., 2013. Use of clopidogrel with or without aspirin in patients taking oral anticoagulant therapy and undergoing percutaneous coronary intervention: an open-label, randomised, controlled trial. Lancet (London, England) 381, 1107-1115. https://doi.org/10.1016/S0140-6736(12)62177-1

Ferreira, A.G., Trotter, S.E., König, B., Decourt, L. V, Fox, K., Olsen, E.G., 1991. Myocardial bridges: morphological and functional aspects. Heart 66, 364-367.

Henriques, J.P.S., Zijlstra, F., Ottervanger, J.P., De Boer, M.-J., Van’T Hof, A.W.J., Hoorntje, J.C.A., Suryapranata, H., 2002. Incidence and clinical significance of distal embolization during primary angioplasty for acute myocardial infarction. Eur. Heart J. 23, 1112-1117.

Iwasaki, Y., Nishida, K., Kato, T., Nattel, S., 2011. Atrial fibrillation pathophysiology: implications for management. Circulation 124, 2264-2274.

Kolodgie, F.D., Virmani, R., Finn, A. V, Romero, M.E., 2015. Embolic myocardial infarction as a consequence of atrial fibrillation: a prevailing disease of the future.

Lee, M.S., Chen, C.-H., 2015. Myocardial bridging: an up-to-date review. J. Invasive Cardiol. $27,521$.

Lopes, R.D., Heizer, G., Aronson, R., Vora, A.N., Massaro, T., Mehran, R., Goodman, S.G., Windecker, S., Darius, H., Li, J., 2019. Antithrombotic therapy after acute coronary syndrome or PCI in atrial fibrillation. N. Engl. J. Med. 380, 1509-1524.

Mega, J.L., Braunwald, E., Wiviott, S.D., Bassand, J.-P., Bhatt, D.L., Bode, C., Burton, P., Cohen, M., CookBruns, N., Fox, K.A.A., 2012. Rivaroxaban in patients with a recent acute coronary syndrome. N. Engl. J. Med. 366, 9-19.

Möhlenkamp, S., Hort, W., Ge, J., Erbel, R., 2002. Update on Myocardial Bridging. Circulation 106, 26162622. https://doi.org/10.1161/01.CIR.0000038420.14867.7A

Murtaza, G., Mukherjee, D., Gharacholou, S.M., Nanjundappa, A., Lavie, C.J., Khan, A.A., Shanmugasundaram, M., Paul, T.K., 2020. An updated review on myocardial bridging. Cardiovasc. Revascularization Med. 21, 1169-1179.

Noble, J., Bourassa, M.G., Petitclerc, R., Dyrda, I., 1976. Myocardial bridging and milking effect of the left anterior descending coronary artery: normal variant or obstruction? Am. J. Cardiol. 37, 993-999.

Ray, B., Keyrouz, S.G., 2014. Management of anticoagulant-related intracranial hemorrhage: an evidence-based review. Crit. Care 18, 1-13.

Soliman, E.Z., Safford, M.M., Muntner, P., Khodneva, Y., Dawood, F.Z., Zakai, N.A., Thacker, E.L., Judd, S., Howard, V.J., Howard, G., Herrington, D.M., Cushman, M., 2014. Atrial Fibrillation and the Risk of Myocardial Infarction. JAMA Intern. Med. 174, 107-114.

https://doi.org/10.1001/jamainternmed.2013.11912

Steiner, T., 2011. Resumption of oral anticoagulation after warfarin-associated intracerebral hemorrhage: yes. Stroke 42, 3661-3662.

Thygesen, K., Alpert, J.S., Jaffe, A.S., Simoons, M.L., Chaitman, B.R., White, H.D., Thygesen, K., Alpert, J.S., White, H.D., Jaffe, A.S., Katus, H.A., Apple, F.S., Lindahl, B., Morrow, D.A., Chaitman, B.R., 
Clemmensen, P.M., Johanson, P., Hod, H., Underwood, R., Bax, J.J., Bonow, R.O., Pinto, F., Gibbons, R.J., Fox, K.A., Atar, D., Newby, L.K., Galvani, M., Hamm, C.W., Uretsky, B.F., Steg, P.G., Wijns, W., Bassand, J.-P., Menasche, P., Ravkilde, J., Ohman, E.M., Antman, E.M., Wallentin, L.C., Armstrong, P.W., Simoons, M.L., Januzzi, J.L., Nieminen, M.S., Gheorghiade, M., Filippatos, G., Luepker, R. V, Fortmann, S.P., Rosamond, W.D., Levy, D., Wood, D., Smith, S.C., Hu, D., Lopez-Sendon, J.-L.,

Robertson, R.M., Weaver, D., Tendera, M., Bove, A.A., Parkhomenko, A.N., Vasilieva, E.J., Mendis, S., 2012. Third Universal Definition of Myocardial Infarction. J. Am. Coll. Cardiol. 60, 1581 LP - 1598. https://doi.org/10.1016/j.jacc.2012.08.001

Tsujita, K., Maehara, A., Mintz, G.S., Doi, H., Kubo, T., Castellanos, C., Liu, J., Yang, J., Oviedo, C., FranklinBond, T., 2009. Impact of myocardial bridge on clinical outcome after coronary stent placement. Am. J. Cardiol. 103, 1344-1348.

Wu, Q., Xu, Z., 2007. Surgical treatment of myocardial bridging: report of 31 cases. Chin. Med. J. (Engl). 120, 1689-1693.

Yuan, S.-M., 2016. Myocardial bridging. Brazilian J. Cardiovasc. Surg. 31, 60-62. 